\title{
USF1 gene variants contribute to metabolic traits in men in a longitudinal 32-year follow-up study
}

\author{
K. Auro • K. Kristiansson • B. Zethelius • C. Berne • \\ L. Lannfelt • M.-R. Taskinen • M. Jauhiainen • \\ M. Perola • L. Peltonen • A.-C. Syvänen
}

Received: 21 June 2007 / Accepted: 12 October 2007 / Published online: 21 December 2007

(C) Springer-Verlag 2007

\begin{abstract}
Aims/hypothesis Genetic variants of upstream transcription factor 1 (USF1) have previously been associated with dyslipidaemias in family studies. Our aim was to further address the role of USF1 in metabolic syndrome and cardiovascular traits at the population level in a large Swedish male cohort $(n=2,322)$ with multiple measurements for risk factors during 32 years of follow-up.
\end{abstract}

Electronic supplementary material The online version of this article (doi:10.1007/s00125-007-0892-9) contains supplementary material, which is available to authorised users.

K. Auro $\cdot$ K. Kristiansson $\cdot$ M. Jauhiainen $\cdot$

M. Perola $\cdot$ L. Peltonen $(\triangle)$

Department of Molecular Medicine,

National Public Health Institute,

Biomedicum, Haartmaninkatu 8,

00290 Helsinki, Finland

e-mail: Leena.Peltonen@ktl.fi

B. Zethelius • L. Lannfelt

Department of Public Health and Caring Sciences,

Uppsala University,

Uppsala, Sweden

C. Berne $\cdot$ A.-C. Syvänen

Department of Medical Sciences, Uppsala University,

Uppsala, Sweden

\section{M.-R. Taskinen}

Department of Medicine,

Helsinki University Central Hospital,

Helsinki, Finland

\section{Perola $\cdot$ L. Peltonen}

Department of Medical Genetics, University of Helsinki,

Helsinki, Finland

\section{Peltonen}

The Broad Institute of MIT and Harvard,

Boston, MA, USA
Methods Participants, born in 1920-1924, were examined at 50,60, 70 and 77 years of age. The follow-up period for cardiovascular events was 1970-2002. We genotyped three haplotype tagging polymorphisms capturing the major allelic variants of USF1.

Results SNP rs2774279 was associated with the metabolic syndrome. The minor allele of rs 2774279 was less common among individuals with metabolic syndrome than among healthy controls $[p=0.0029$ when metabolic syndrome was defined according to the National Cholesterol Education Program Adult Treatment Panel III; $p=0.0073$ when defined according to the International Diabetes Federation (IDF)]. The minor allele of rs2774279 was also associated with lower BMI, lower fasting glucose values and higher HDL-cholesterol concentrations in longitudinal analyses. With SNP rs2073658, a borderline association with metabolic syndrome was observed $(p=0.036$, IDF), the minor allele being the risk-increasing allele. The minor allele of rs2073658 also associated with higher total and LDL-cholesterol, apolipoprotein B-100 and lipoprotein(a) concentrations in longitudinal analyses. Importantly, these trends with respect to the allelic variants prevailed throughout the follow-up time of three decades.

Conclusions/interpretation Our results suggest that USF1 variants associate with the metabolic syndrome at population level and influence the cardiovascular risk factors throughout adulthood in a consistent, longitudinal manner.

Keywords Cardiovascular diseases · Epidemiology . Genetics · Lipids · Metabolic syndrome · Syndrome X

\author{
Abbreviations \\ Apo apolipoprotein \\ ATPIII National Cholesterol Education Program Adult \\ Treatment Panel III
}


CVD cardiovascular disease

EGIR European Group for the Study of Metabolic Syndrome

FCHL familial combined hyperlipidaemia

HOMA homeostasis assessment model

ICD International Classification of Diseases

IDF International Diabetes Federation

Lp(a) lipoprotein(a)

SNP single nucleotide polymorphism

ULSAM Uppsala Longitudinal Study of Adult Men

\section{Introduction}

Metabolic syndrome is characterised by various definitions [1-4] as different combinations of elevated triacylglycerol, low HDL-cholesterol, elevated fasting glucose, insulin resistance, hypertension and central obesity. Metabolic syndrome predisposes to type 2 diabetes, cardiovascular (CVD) events and mortality [5-8]. Upstream transcription factor 1 (USF1) regulates the expression of more than 40 genes, many of them participating in body energy and lipid metabolism [6]. USF1 has been associated with familial combined hyperlipidaemia (FCHL), a hereditary condition that overlaps with metabolic syndrome. Recent studies on Finnish [9], Mexican [10] and Dutch [11] FCHL families and USF 1 have indicated that $U S F 1$ variants associate with FCHL, especially in men. The role of USF1 in metabolic syndrome and type 2 diabetes has also been studied; a Chinese study reported positive results [12] but no association was seen in French families [13]. Association of USF1 with triacylglycerol or serum total cholesterol or both has been reported in Mexican, American and Finnish studies $[9,10,14]$. At the population level, however, no association of USF1 with type 2 diabetes, CVD or metabolic syndrome has been reported in men $[12,13,15$, 16], although a mixed European study sample showed association with serum lipid and glucose parameters and BMI in healthy men with a family history of CVD [17].

This study aimed to further address the role of USF1 in metabolic syndrome and cardiovascular traits. We hypothesised that USF1 variants influence serum lipids and glucose parameters and potentially contribute to the lifetime risk of metabolic syndrome and CVD in men also at the population level. Our results imply that USF1 variants associate with metabolic syndrome and influence its component traits throughout adulthood.

\section{Methods}

Study sample and measurements The Uppsala Longitudinal Study of Adult Men (ULSAM) invited all men in Uppsala
County, Sweden who were born in 1920-1924 to take part in investigations four times throughout adulthood, at the ages of 50 (1970-1973), 60 (1980-1984), 70 (1991-1995) and 77 (1997) years. In each survey, all participants underwent a medical interview and completed a selfquestionnaire. All four surveys included measurements of body anthropometrics, cholesterol levels (total cholesterol, HDL-cholesterol and triacylglycerol), fasting glucose and blood pressure. Apolipoprotein (Apo) A-I, ApoB-100 and lipoprotein(a) $[\mathrm{Lp}(\mathrm{a})]$ were also measured at the first (50 years) and the third (70 years) survey. Table 1 gives the measurements recorded in the different surveys. Most participants had a white ethnic background.

Blood pressure was measured in the supine position after $10 \mathrm{~min}$ of rest. Weight and height were measured for every participant in all surveys. Waist circumference was measured only at baseline in those born between 1920 and 1921, and in every participant in the later three surveys. Lipid samples drawn in 1970-1973 were stored in liquid nitrogen until analysed in 1981 or 1988 (all apolipoproteins). Values were adjusted using the conversion factors 1.06 for LDLcholesterol and total cholesterol, 0.9 for triacylglycerol and 1.17 for HDL-cholesterol to enable comparison with the Monarch method used in the surveys at ages 70 and 77. LDLcholesterol was calculated with Friedewald's formula at all ages using $4.0 \mathrm{mmol} / \mathrm{l}$ as the cutoff limit for triacylglycerol. The third survey (70 years) included a euglycaemic insulin clamp test.

Cardiovascular events were followed during 1970-2002 from the National Hospital Discharge Register and the National Causes of Death Register (International Classification of Diseases [ICD]-9 codes 410-414 and ICD-10 codes I20-I21, I25 for coronary events, and ICD-9 codes 433-434 and ICD-10 code I63 for ischaemic strokes).

The study cohort and the methods used are described in previous articles [18, 19] and at the ULSAM website (http://www.pubcare.uu.se/ULSAM/index.htm). The Ethics Committee of the Medical Faculty of Uppsala University, Sweden, approved the study. All participants gave informed consent.

DNA extraction and genotyping Blood for DNA sampling was drawn in the third survey from 1,150 individuals. DNA from these samples was extracted with a standard salting-out procedure. In addition, DNA from paraffin block samples collected from about 500 individuals who died during followup before the third survey was extracted from thin sections of paraffin-embedded tissues using proteinase $\mathrm{K}$ digestion and QIAamp DNA minikits (Qiagen, Valencia, CA, USA).

A single-base primer extension assay with fluorescence polarisation detection [20] was used for genotyping, with reagents from Perkin Elmer Life Sciences (Boston, MA, USA). For the blood samples $(n=1,150)$, the genotyping 
Table 1 Characteristics of the ULSAM cohort: numbers for CVD events, metabolic syndrome and mortality are cumulative from the baseline

\begin{tabular}{|c|c|c|c|c|c|}
\hline & $\begin{array}{l}\text { Survey I, } 50 \text { years, } \\
1970-1973 \\
(n=2,322)\end{array}$ & $\begin{array}{l}\text { Survey II, } 60 \text { years, } \\
1981-1984 \\
(n=1,860)\end{array}$ & $\begin{array}{l}\text { Survey III, } 70 \text { years, } \\
1991-1995 \\
(n=1,221)\end{array}$ & $\begin{array}{l}\text { Survey IV, } 77 \text { years, } \\
1997 \\
(n=839)\end{array}$ & $\begin{array}{l}\text { End of follow-up } \\
2002\end{array}$ \\
\hline CVD events $^{\mathrm{a}}$ & 14 & 134 & 373 & 518 & 701 \\
\hline $\begin{array}{l}\text { Metabolic syndrome } \\
\text { ATPIII }^{\mathrm{b}}\end{array}$ & 425 & 662 & 878 & 969 & - \\
\hline Total mortality & - & 98 & 442 & 748 & 1,078 \\
\hline Ineligible & - & 94 & 219 & 176 & - \\
\hline Measurements & $\begin{array}{l}\text { Questionnaire } \\
\text { Cholesterol }^{\mathrm{c}} \\
\text { Blood pressure } \\
\text { Fasting glucose } \\
\text { BMI } \\
\text { ApoA-I, ApoB-100 } \\
\text { IVGTT }\end{array}$ & $\begin{array}{l}\text { Questionnaire } \\
\text { Cholesterol }^{\mathrm{c}} \\
\text { Blood pressure } \\
\text { Fasting glucose } \\
\text { BMI, waist grid } \\
\text { IVGTT }\end{array}$ & $\begin{array}{l}\text { Questionnaire } \\
\text { Cholesterol }^{\mathrm{c}} \\
\text { Blood pressure } \\
\text { Fasting glucose } \\
\text { BMI, waist grid } \\
\text { ApoA-1, ApoB-100 } \\
\text { Insulin clamp } \\
\text { Blood samples for DNA }\end{array}$ & $\begin{array}{l}\text { Questionnaire } \\
\text { Cholesterol }^{\mathrm{c}} \\
\text { Blood pressure } \\
\text { Fasting glucose } \\
\text { Waist grid }\end{array}$ & \\
\hline
\end{tabular}

${ }^{a}$ Coronary event or ischaemic stroke during follow-up. Nine persons with baseline CVD (50 years of age) had another event during follow-up

${ }^{\mathrm{b}}$ ATPIII criteria (male-specific) used. Individuals with missing values excluded at each time point

${ }^{\mathrm{c}}$ Total cholesterol, HDL-cholesterol, triacylglycerol

call rate was $95-99 \%$ with $100 \%$ reproducibility in 300 genotype comparisons $(9 \%)$ between independent experiments. Of the 500 paraffin samples, only 158 gave acceptable genotyping results. Thus, data from only these 158 samples were included in the analyses. Healthy Swedish blood donors $(n=98)$ were used as a control group for the allele frequencies in the ULSAM cohort.

We genotyped four single-nucleotide polymorphisms (SNPs) at the USF1 gene locus (rs2774279, rs2516839, rs2073658 and rs3737787), covering the three most common haplotype bins (SeattleSNPs database, European descent [21]). rs2774279 was in substantial linkage disequilibrium with rs2516839 in a separate, Finnish family sample (SeattleSNPs bin 7, data not shown). rs2073658 and rs3737787 were in full linkage disequilibrium $\left(r^{2}=1\right)$ in the ULSAM sample with 1,150 individuals, and rs3737787 was therefore excluded. rs2073658 is located in intron 7 and rs2516839 in an untranslated exon of USF1. rs2774279 in the promoter area of $U S F 1$ gene is in turn located within a novel gene ARHGAP30. Figure 1 illustrates the linkage disequilibrium pattern between the SNPs.

Metabolic syndrome We used four different definitions for metabolic syndrome, with sex- and ethnic-specific values: those of the National Cholesterol Education Program Adult Treatment Panel III (NCEP/ATPIII [2]); the International Diabetes Federation (IDF [1]), WHO (excluding microalbuminuria) [3]; and the European Group for the Study of Metabolic Syndrome (EGIR; [4]). Because of the lack of waist measurements, BMI was used instead of waist circumference as a criterion in IDF and ATPIII at baseline (correspondence of BMI $29.4 \mathrm{~kg} / \mathrm{m}^{2}$ for waist circumference $102 \mathrm{~cm}$ and BMI $27.0 \mathrm{~kg} / \mathrm{m}^{2}$ for waist circumference $94 \mathrm{~cm}[8])$. The WHO and EGIR definitions were used at 70 years. To exclude bias in the longitudinal and variance analyses at the age of 70 years, metabolic syndrome was defined without HDL-cholesterol as a criterion when analysing HDL-cholesterol levels, and accordingly with triacylglycerol, waist circumference and fasting glucose.
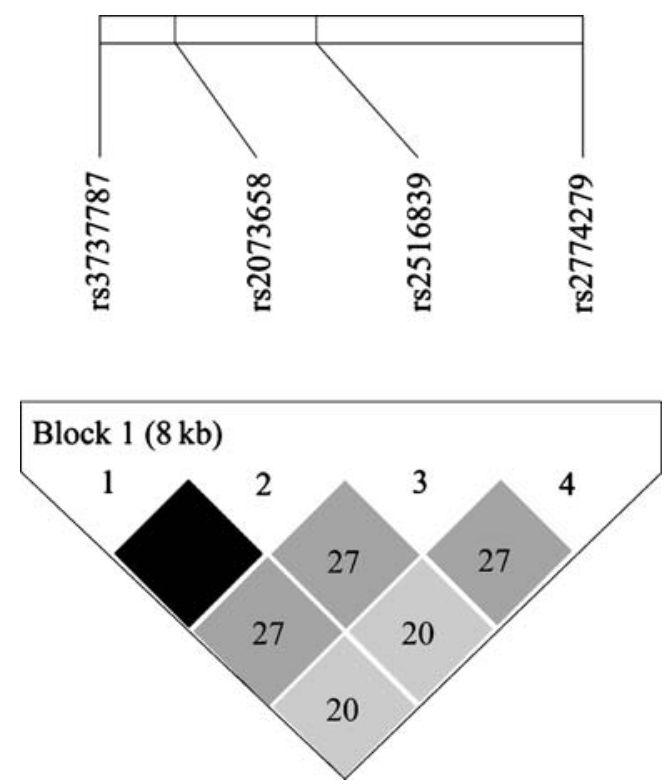

Fig. 1 Linkage disequilibrium pattern of SNPs genotyped in the ULSAM study sample with Haploview 3.2 
Statistical analysis The data set was first analysed as a whole population cohort and then divided into three subgroups: CVD patients, metabolic syndrome patients, and healthy participants free of these conditions. The rationale for this division was the assumption that the impact of USF1 variants on the traits analysed relates to disease status $[15,17]$. We estimated haplotypes [electronic supplementary material (ESM) Table 1] with Haploview 3.2 [22] and PHASE 2.1.1 [23]; 40 separate runs were performed and compared with ensure reliable estimation. All other analyses were performed with SAS v8.2 and SAS 9.1.3 for Windows (SAS, Cary, NC, USA). The analyses were performed assuming dominant inheritance, based on previous reports suggesting dominant inheritance for the USF1 variants $[9,10]$ and in order to gain power.

Statistical analyses were performed in four parts. First, the SNP allele frequencies were compared between CVD and metabolic syndrome cases and healthy controls with Pearson's $\chi^{2}$ test. Second, the risk of CVD events was estimated with Cox univariate and multivariate proportional hazards models [24]. The multivariate model was adjusted for age, diabetes, hypertension, BMI, smoking and total cholesterol/HDL-cholesterol ratio at baseline. Third, longitudinal measurements within individuals from the four surveys were analysed with a longitudinal linear model (PROC MIXED REPEATED of SAS). Longitudinal analyses measure the change in quantitative traits between different gene variants with mixed longitudinal models. Longitudinal models assess the degree and direction of variation in longitudinally measured variables over the time points (the four surveys) in the different analysis groups (here, different gene alleles) as a linear function of time. Mixed longitudinal models are better than the repeated general linear model for data in which there are differences in the sizes of analysis groups; here, in the haplotype distribution between the study participants. The model was corrected for BMI. Finally, we performed cross-sectional analysis at the age of 70 years to compare different metabolic syndrome definitions using the general linear model.

Outliers ( \pm 4 SD) were removed before we analysed the quantitative traits. To achieve normality, LDL-cholesterol, triacylglycerol, BMI, waist circumference grid, and systolic and diastolic blood pressures were log-transformed, and HDL-cholesterol, Lp(a), fasting glucose, homeostasis assessment model (HOMA) beta cell, and the clamp variables were corrected using a rank procedure. Individuals using lipid $(n=166)$, antihypertensive $(n=466)$ or diabetes $(n=$ 196) medication were excluded from the analyses for these variables. To assess multiple testing, a threshold for statistical significance was calculated with 1,000 random permutations: SNP analyses were performed after randomly shuffling the genotypes in the original data file, repeating these two steps 1,000 times, and the significance level, corrected for multiple testing, was calculated as the fifth percentile limit. One thousand independent permutations were performed for each of the variables in all the separate analyses, resulting in variable- and analysis-specific thresholds for significance $(p<0.05)$.

\section{Results}

Prevalence of metabolic syndrome and CVD in the study sample At baseline, 2,322 men aged 50 years participated in the clinical investigations (1970-1973). At 60, 70 and 77 years the number of men investigated was 1,860, 1,221 and 839 , respectively. By the end of follow-up in 2002, 1,078 participants $(46 \%)$ had died. During the whole $32-$ year follow-up period, 701 men suffered from a coronary event or an ischaemic stroke. Table 1 provides the characteristics of the study cohort and measurements relevant to this study performed in different surveys.

We used ATPIII and IDF definitions to assess the lifelong risk of metabolic syndrome, and additionally the EGIR and WHO criteria at 70 years. The overall prevalence of metabolic syndrome was alarmingly high, 41.7\% (969 of the 2,322 participants) with the ATPIII definition and $39.0 \%$ (905 of the 2,322 participants) with the IDF definition. The ATPIII definition had best overlap with CVD (33.9\%). With the other three definitions, the coprevalence of CVD was 29.4-29.8\% (ESM Table 2). Metabolic syndrome at baseline was a strong predictor of future CVD: $43.1 \%$ of those fulfilling the metabolic syndrome criteria (ATPIII) at baseline had a cardiovascular event in the next 30 years of life (ESM Table 2). Metabolic syndrome as a predictor of CVD in the ULSAM cohort has been analysed in detail previously [8].

Serum total cholesterol, triacylglycerol and LDLcholesterol concentrations declined with age (Table 2). BMI, waist circumference, blood pressure and fasting glucose levels increased as a function of age (Table 2). These trends were similar in all participants and in those participating in all four surveys.

USF1 haplotypes and minor allele frequencies The minor alleles of the three SNPs analysed tagged the three common haplotypes of USF1. In PHASE haplotype estimations, other haplotypes than these three were each seen only in two participants $(0.3 \%$; ESM Table 1). Further, the haplotype estimation probabilities for the three common haplotypes were 1.00 for each participant. We therefore concluded that the SNP allele carrierships corresponded nearly perfectly to carriership of the haplotype tagged by the SNP, and separate haplotype analyses were thus excluded from the study. All the SNPs had a minor allele frequency (minor allele frequency) of $>0.29$ (Table 3) and 
Table 2 Characteristics (mean \pm SD) of the ULSAM cohort

\begin{tabular}{|c|c|c|c|c|}
\hline \multirow[t]{2}{*}{ Variable } & \multicolumn{4}{|l|}{ Age (years) } \\
\hline & $\begin{array}{l}50 \\
(n=2,322)\end{array}$ & $\begin{array}{l}60 \\
(n=1,860)\end{array}$ & $\begin{array}{l}60 \\
(n=1,221)\end{array}$ & $\begin{array}{l}60 \\
(n=839)\end{array}$ \\
\hline \multirow[t]{2}{*}{ Total cholesterol $(\mathrm{mmol} / \mathrm{l})^{\mathrm{a}}$} & $6.87 \pm 1.31$ & $6.31 \pm 1.10$ & $5.82 \pm 1.00$ & $5.48 \pm 0.96$ \\
\hline & $6.78 \pm 1.29^{\mathrm{e}}$ & $6.25 \pm 1.07^{\mathrm{e}}$ & $5.82 \pm 0.98^{\mathrm{e}}$ & $5.48 \pm 0.96^{\mathrm{e}}$ \\
\hline \multirow[t]{2}{*}{ LDL-cholesterol $(\mathrm{mmol} / \mathrm{l})^{\mathrm{a}}$} & $5.26 \pm 1.24$ & $4.28 \pm 0.96$ & $3.90 \pm 0.89$ & $3.56 \pm 0.84$ \\
\hline & $5.19 \pm 1.26^{\mathrm{e}}$ & $4.15 \pm 0.88^{\mathrm{e}}$ & $3.87 \pm 0.88^{\mathrm{e}}$ & $3.47 \pm 0.70^{\mathrm{e}}$ \\
\hline \multirow[t]{2}{*}{ Triacylglycerol $(\mathrm{mmol} / \mathrm{l})^{\mathrm{a}}$} & $1.93 \pm 1.22$ & $1.85 \pm 1.01$ & $1.45 \pm 0.78$ & $1.36 \pm 0.68$ \\
\hline & $1.78 \pm 0.85^{\mathrm{e}}$ & $1.7 \pm 0.83^{\mathrm{e}}$ & $1.41 \pm 0.78^{\mathrm{e}}$ & $1.36 \pm 0.68^{\mathrm{e}}$ \\
\hline \multirow[t]{2}{*}{ HDL-cholesterol $(\mathrm{mmol} / \mathrm{l})^{\mathrm{a}}$} & $1.36 \pm 0.40$ & $1.19 \pm 0.34$ & $1.28 \pm 0.35$ & $1.32 \pm 0.33$ \\
\hline & $1.37 \pm 0.37^{\mathrm{e}}$ & $1.20 \pm 0.32^{\mathrm{e}}$ & $1.3 \pm 0.35^{\mathrm{e}}$ & $1.32 \pm 0.33^{\mathrm{e}}$ \\
\hline \multirow[t]{2}{*}{$\operatorname{Lp}(\mathrm{a})(\mu \mathrm{mol} / 1)^{\mathrm{a}}$} & $0.61 \pm 0.76$ & - & $0.76 \pm 0.90$ & - \\
\hline & $0.58 \pm 0.75^{\mathrm{e}}$ & - & $0.76 \pm 0.89^{\mathrm{e}}$ & - \\
\hline \multirow[t]{2}{*}{ ApoA-I $(\mathrm{g} / \mathrm{l})^{\mathrm{a}}$} & $1.43 \pm 0.25$ & - & $1.28 \pm 0.23$ & - \\
\hline & $1.44 \pm 0.23^{\mathrm{e}}$ & - & $1.30 \pm 0.23^{\mathrm{e}}$ & - \\
\hline \multirow[t]{2}{*}{ ApoB-100 (g/l) } & $1.24 \pm 0.28$ & - & $1.03 \pm 0.23$ & - \\
\hline & $1.21 \pm 0.27^{\mathrm{e}}$ & - & $1.02 \pm 0.22^{\mathrm{e}}$ & - \\
\hline \multirow[t]{2}{*}{ Fasting glucose $(\mathrm{mmol} / \mathrm{l})^{\mathrm{b}}$} & $4.96 \pm 0.61$ & $4.81 \pm 0.58$ & $5.36 \pm 0.58$ & $5.48 \pm 0.58$ \\
\hline & $4.92 \pm 0.56^{\mathrm{e}}$ & $4.79 \pm 0.50^{\mathrm{e}}$ & $5.38 \pm 0.59^{\mathrm{e}}$ & $5.48 \pm 0.58^{\mathrm{e}}$ \\
\hline \multirow[t]{2}{*}{ HOMA beta cell index ${ }^{c}$} & $9.98 \pm 10.22$ & $5.63 \pm 3.64$ & $7.16 \pm 5.68$ & - \\
\hline & $9.65 \pm 13.79^{\mathrm{e}}$ & $5.44 \pm 3.54^{\mathrm{e}}$ & $6.97 \pm 4.42^{\mathrm{e}}$ & - \\
\hline \multirow[t]{2}{*}{ Blood pressure $(\mathrm{mmHg})^{\mathrm{d}}$} & $132 / 83 \pm 17 / 11$ & $140 / 86 \pm 19 / 9$ & $144 / 82 \pm 17 / 9$ & $149 / 80 \pm 21 / 10$ \\
\hline & $130 / 82 \pm 15 / 10^{\mathrm{e}}$ & $138 / 86 \pm 18 / 9^{\mathrm{e}}$ & $143 / 82 \pm 17 / 9^{\mathrm{e}}$ & $149 / 80 \pm 21 / 10^{\mathrm{e}}$ \\
\hline \multirow[t]{2}{*}{ BMI $\left(\mathrm{kg} / \mathrm{m}^{2}\right)$} & $25.03 \pm 3.22$ & $25.49 \pm 3.28$ & $26.30 \pm 3.43$ & - \\
\hline & $24.78 \pm 2.87^{\mathrm{e}}$ & $25.32 \pm 3.04^{\mathrm{e}}$ & $26.30 \pm 3.29^{\mathrm{e}}$ & - \\
\hline \multirow[t]{2}{*}{ Waist circumference $(\mathrm{cm})$} & $87.86 \pm 8.84$ & $90.99 \pm 10.03$ & $94.82 \pm 9.65$ & $95.60 \pm 9.86$ \\
\hline & $86.38 \pm 8.55^{\mathrm{e}}$ & $89.54 \pm 9.85^{\mathrm{e}}$ & $94.62 \pm 9.26^{\mathrm{e}}$ & $95.60 \pm 9.86^{\mathrm{e}}$ \\
\hline
\end{tabular}

${ }^{\mathrm{a}}$ Individuals using lipid-lowering medication removed $(n=166)$

${ }^{\mathrm{b}}$ Individuals using glucose-lowering medication removed $(n=196)$

${ }^{\mathrm{c}}$ Fasting insulin $(\mu \mathrm{U} / \mathrm{ml}) /[$ fasting glucose $(\mathrm{mmol} / \mathrm{l})-3.5]$ (to convert serum insulin in $\mathrm{pmol} / \mathrm{l}$ to $\mu \mathrm{U} / \mathrm{ml}$, divide the value by 6.945 )

${ }^{\mathrm{d}}$ Individuals using antihypertensive medication removed $(n=466)$

${ }^{\mathrm{e}}$ Characteristics of a subsample participating in all four surveys $(n=839)$

were in Hardy-Weinberg equilibrium at the $p>0.05$ level (data not shown). To estimate the possible bias caused by late DNA sampling, we compared minor allele frequencies in the whole ULSAM cohort (alive at the 70 year survey), in paraffin-embedded tissue (representing those who died before the 70 year survey) and in 98 Swedish blood donors (representing the general Swedish population). No significant differences in allele frequencies were observed (ESM Table 3).

Table 3 Minor allele frequencies among the metabolic syndrome (metabolic syndrome) group and the healthy controls (free of metabolic syndrome and CVD)

\begin{tabular}{lllll}
\hline SNP & rs2073658 & rs2774279 & rs2516839 $^{\text {Significance level }^{\mathrm{a}}}$ \\
\hline Metabolic syndrome, ATPIII, 70 years $(n=384)$ & 0.33 & $0.27^{\mathrm{e}}$ & 0.39 & 0.0167 \\
Metabolic syndrome, IDF, 70 years $(n=435)$ & $0.35^{\mathrm{c}}$ & $0.28^{\mathrm{d}}$ & 0.38 & 0.0173 \\
Metabolic syndrome, EGIR, 70 years $(n=269)$ & 0.34 & 0.30 & 0.36 & 0.0146 \\
Metabolic syndrome, WHO, 70 years $(n=413)$ & 0.33 & $0.29^{\mathrm{c}}$ & 0.39 & 0.0211 \\
Metabolic syndrome, ATPIII, lifelong $(n=969)^{\mathrm{b}}$ & 0.32 & $0.29^{\mathrm{d}}$ & 0.39 & 0.0179 \\
Metabolic syndrome, IDF, lifelong $(n=905)^{\mathrm{b}}$ & 0.32 & $0.29^{\mathrm{d}}$ & 0.39 & 0.0223 \\
Healthy controls $(n=998)$ & 0.30 & 0.33 & 0.38 & \\
\hline
\end{tabular}

${ }^{a}$ Multiple testing corrected significance level for $\chi^{2}$ test for allelic difference between cases and the controls

${ }^{\mathrm{b}}$ Lifelong: metabolic syndrome diagnosed at one or more surveys

${ }^{\mathrm{c}} p<0.05,{ }^{\mathrm{d}} p<0.01,{ }^{\mathrm{e}} p<0.001$ for difference in minor allele frequencies between metabolic syndrome group and healthy controls 
Association of SNPS with metabolic syndrome and cardiovascular disease The minor allele of the SNP rs2774279 was significantly less common in participants with metabolic syndrome than in the healthy controls free of metabolic syndrome and CVD (Table 3). This trend was consistent among the ATPIII, IDF and WHO definitions of metabolic syndrome, but not the EGIR definition (Table 3). A suggestive association of the metabolic syndrome with the SNP rs2073658 was seen: the minor allele of rs2073658 was more common in the metabolic syndrome groups defined with the IDF definition at 70 years than in the healthy controls $(p<0.05$; Table 3$)$. None of the SNPs showed a direct association with CVD when allele frequencies were compared between cases and controls or in the Cox proportional hazards model (data not shown). The results were similar when analysing only the DNA samples extracted from whole blood $(n=1,150)$.

Association of the SNPS with metabolic syndrome components Longitudinal measurements within individuals were assessed in longitudinal linear analyses performed in four separate groups: the whole study cohort; CVD patients; individuals with metabolic syndrome; and healthy controls free of CVD and metabolic syndrome. To detect the direction of change in longitudinal variables, we calculated mean values of metabolic syndrome components at different ages, following the same data division at each age.

The results of the longitudinal analyses supported the putative protective role of the minor allele of the SNP rs2774279 and the putative risk-increasing role of the minor allele of the SNP rs2073658. The minor allele of rs 2774279 was associated with lower BMI (in the whole cohort) and lower fasting glucose concentration (in CVD patients; Table 4). Trends for lower BMI in the CVD patients, smaller waist grid (in the whole cohort) and higher HDLcholesterol concentrations (in the whole cohort, the CVD patients and the metabolic syndrome group) were also seen in carriers of the minor allele of rs2774279. The minor allele of rs2073658 was associated with higher total cholesterol and ApoB-100 values in the metabolic syndrome group (ATPIII definition). Trends for higher LDLcholesterol (in the metabolic syndrome group) and higher $\mathrm{Lp}$ (a) concentrations (in the CVD group and healthy controls) were also observed with this variant. Carriers of the minor allele of rs2516839 had a trend for lower BMI in the CVD patients. Table 4 and ESM Tables 4, 5, 6, 7 illustrate the results of longitudinal analyses.

Cross-sectional analysis: 70 year survey To compare the different clinical definitions of metabolic syndrome we performed a separate association analysis as a cross-section of the data at 70 years. The results further supported the findings from the longitudinal analyses, suggesting a protective role for the minor allele of the SNP rs2774279: carriers of the minor allele had higher mean HDL ( $p=$ 0.0232 to $p=0.0603)$ and lower mean $\operatorname{Lp}(\mathrm{a})(p=0.0052$ to $p=0.0692$ ) values than the carriers of other haplotypes, independently of the metabolic syndrome definition used. Furthermore, the minor allele of rs2774279 was associated with lower BMI ( $p=0.0099$ for the whole cohort; $p=0.0346$

Table 4 Association ( $p$ values) of the USF1 SNPs with the longitudinally measured cardiovascular risk factors in longitudinal linear analysis (PROC MIXED of SAS v. 8)

\begin{tabular}{|c|c|c|c|c|c|c|c|c|c|c|c|c|}
\hline \multirow{2}{*}{$\begin{array}{l}\text { Longitudinal } \\
\text { variable }\end{array}$} & \multicolumn{4}{|c|}{ rs 2073658} & \multicolumn{4}{|c|}{ rs 2774279} & \multicolumn{4}{|c|}{ rs 2516839} \\
\hline & All & $\begin{array}{l}\text { Metabolic } \\
\text { syndrome }^{\mathrm{a}}\end{array}$ & $\mathrm{CVD}^{\mathrm{b}}$ & Healthy $^{c}$ & All & $\begin{array}{l}\text { Metabolic } \\
\text { syndrome }^{\mathrm{a}}\end{array}$ & $\mathrm{CVD}^{\mathrm{b}}$ & Healthy $^{\mathrm{c}}$ & All & $\begin{array}{l}\text { Metabolic } \\
\text { syndrome }^{\mathrm{a}}\end{array}$ & $\mathrm{CVD}^{\mathrm{b}}$ & Healthy $^{\mathrm{c}}$ \\
\hline BMI & & & & & $0.0188^{\mathrm{e}}$ & & 0.0484 & & & & 0.0460 & \\
\hline Waist circumference & & & & & 0.0685 & & & & & & & \\
\hline Total cholesterol $^{\mathrm{d}}$ & & $0.0142^{\mathrm{e}}$ & & & & & & & & & & \\
\hline LDL-cholesterol $^{\mathrm{d}}$ & & 0.0242 & & & & & & & & & & \\
\hline HDL-cholesterol $^{\mathrm{d}}$ & & & & & 0.0620 & 0.0242 & 0.0611 & & & & & \\
\hline ApoB- $100^{\text {d }}$ & & $0.0136^{\mathrm{e}}$ & & & & & & & & & & \\
\hline ApoA-I ${ }^{\mathrm{d}}$ & & & & & & & & & & & & \\
\hline $\operatorname{Lp}(a)^{d}$ & & & 0.0232 & 0.0277 & & & & & & & & \\
\hline Fasting glucose & & & & & 0.0397 & & $0.0116^{\mathrm{e}}$ & & & & & \\
\hline HOMA beta cell ${ }^{\mathrm{e}}$ & & & & & & & 0.0532 & & & & & \\
\hline
\end{tabular}

Analysis of SNPs assumed dominant inheritance

${ }^{\text {a }}$ Metabolic syndrome by ATPIII criteria

${ }^{\mathrm{b}}$ Individuals with cardiovascular events

${ }^{\mathrm{c}}$ Individuals with no CVD events or metabolic syndrome

${ }^{\mathrm{d}}$ Individuals with lipid-lowering medication excluded from analysis

${ }^{\mathrm{e}}$ Significant after multiple testing correction as fifth percentile of 1,000 permutations 
for the CVD group), higher HDL-cholesterol concentration ( $p=0.0315$, whole cohort), lower fasting glucose values ( $p=$ 0.0122 , whole cohort), higher mean glucose disposal ( $p=$ 0.0410 , whole cohort) and higher metabolic clearance rate of glucose during the clamp test $(p=0.042$, healthy participants). Carriers of the minor allele of rs2073658 had lower mean diastolic blood pressure in the whole cohort $(p=$ $0.0206)$ and in the CVD group ( $p=0.0360)$. Carriers of the minor allele of rs 2516839 had lower BMI $(p=0.0466)$.

\section{Discussion}

Metabolic syndrome is increasing rapidly in all Western societies. The molecular background of the syndrome remains unknown but the individual traits characterising the condition are strongly hereditary, and several studies have reported clustering of these traits [25, 26]. In our study the prevalence of metabolic syndrome was alarmingly high (41.9\%). Similar figures have been reported from other populations in older age groups for both sexes [27]. Because of the lower cutoff point for fasting glucose in our study (5.6 vs $6.1 \mathrm{mmol} / \mathrm{l}$ ), our figures for metabolic syndrome differ slightly from those previously reported for the ULSAM cohort [8]. Definitions for the metabolic syndrome have changed historically, and it is yet to be established which one of the several criteria best reflects the biological background. We used four different definitions in our analyses within the limits of the information accessible in our surveys.

Our results suggest that $U S F 1$ variants contribute to the risk of metabolic syndrome in men at the population level. We identified both a putative protective variant, the minor allele of rs 2774279 , and a putative risk variant, the minor allele of rs2073658. The results of the metabolic syndrome component trait analyses support the protective and riskincreasing roles of these two variants. The third variant analysed, rs2516839, was not associated with metabolic syndrome. Although $51.1 \%$ (358 out of 701) of the ULSAM CVD patients also had metabolic syndrome, no direct association between CVD and the USF1 variants was observed. The variants showed no association with CVD within the metabolic group either. Metabolic syndrome defined by ATPIII criteria had slightly better overlap with CVD than when the other three definitions were used. Because of the bias created by the sampling of DNA late in life, we were not in a position to assess mortality in this study.

USF1 SNPs have shown association with dyslipidaemias and FCHL in several studies. The risk variants, however, have varied from study to study. Pajukanta et al. [9] reported association of USF1 SNP rs2073658 with triacylglycerol levels in Finnish FCHL families, the major allele being the risk allele. The finding has been replicated in Mexican and Dutch FCHL families [10, 11]. Two population studies not ascertained for any specific endpoint have, however, identified the minor allele of the SNP as the risk variant $[15,16]$. Our study used a population-based sample, not ascertained for any specific diagnosis. We observed a suggestive association of the minor allele of rs2073658 with increased risk of metabolic syndrome, as well as with atherogenic lipid traits in adulthood. The discrepancy in the risk alleles between FCHL families and population samples may point to underlying gene-gene interactions modulating the influence of USF1 on lipid traits. Elevated Lp(a) is known to be associated with CVD risk. The association of USF1 with $\mathrm{Lp}(\mathrm{a})$ has not been established previously. Association of rs2516839 with lower diastolic blood pressure might reflect the effect of USF1 haplotypes on angiotensinogen expression [28].

Putt et al. [17] observed that the association of USF1 variants with LDL-cholesterol concentrations was dependent on BMI. Furthermore, in our recent population basedstudy USF1 variants were associated with CVD risk factors only among patients with CVD and not in a healthy subcohort [15]. Accordingly, in the ULSAM data the effect of the USF1 variants on cardiovascular risk factors was seen especially in individuals having metabolic syndrome or cardiovascular disease or both. These findings may point to gene-gene or gene-environment interactions modifying the influence of USF1 variants on cardiovascular risk factors.

The major strength of this study is its longitudinal and prospective nature. The CVD follow-up time of 32 years is to our knowledge among the longest reported in the literature. The key components of the metabolic syndrome-fasting glucose, serum triacylglycerol and HDL-cholesterol, blood pressure and waist circumference or BMI-were measured at four different time points in the same hospital. Lipid values, obtained by slightly different methods, were corrected to be comparable. No selection bias was detected with the early lipid samples stored in liquid nitrogen before analysis. The possible effect of this storage would increase the variability of the results and thus dilute the association detected in the study, leading to under- rather than overestimation of the risk reported here. We also used a full age cohort of men from the same small geographical area, representing a homogeneous white population. The significance level was corrected for multiple testing. Limitations of this study include the lack of female participants and the loss of individuals from genetic analysis due to late DNA sampling. However, the allele frequencies observed within the whole ULSAM sample, among the subsample of those who died before the age of 70 years, and in the Swedish 
control sample did not differ significantly, nor did the mean values of the lipid and glucose parameters calculated within the whole study sample and among those participating in all four samplings. It is therefore likely that our genotypic sample was reliable.

In conclusion, this is the first population study to have established an association of USF1 gene with the metabolic syndrome, defining both putative risk and protective variants. The accumulation of significant findings in the metabolic and the CVD groups suggests an underlying gene-gene and/or gene-environment interaction. These findings support previous reports $[26,29]$ suggesting that the accumulation of the components defining metabolic syndrome is genetically determined, and provide additional evidence for the role of USF1 as a metabolic and cardiovascular risk factor.

Acknowledgements We wish to thank all the ULSAM study participants. J. Kaprio and T. Hiekkalinna are thanked for their excellent statistical assistance and T. Axelsson and C. Pöntinen for their assistance with genotyping. Genotyping was performed by the SNP Technology Platform at Uppsala University, Sweden (http://www. genotyping.se). This study was supported by Finnish Foundation for Cardiovascular Research, Jenny and Antti Wihuri Foundation, Aarne Koskelo foundation, Knut and Alice Wallenberg Foundation, Ernfors Family Foundation, Sehlander Foundation, Swedish Diabetes Association, the Center of Excellence of Disease Genetics by the Academy of Finland, the Nordic Center of Excellence in Disease Genetics, the GenomEUtwin-project (http://www.genomeutwin.org) under the European Commission 'Quality of Life and Management of the Living Resources' 5th Framework Programme (no. QLG2-CT-2002-01254) and NIH/NHLBI (1R01HL70150-01A1).

Duality of interest The authors declare that there is no duality of interest associated with this manuscript.

\section{References}

1. Alberti KG, Zimmet P, Shaw J (2006) Metabolic syndrome-a new world-wide definition. A Consensus Statement from the International Diabetes Federation. Diabet Med 23:469-480

2. Executive Summary of The Third Report of The National Cholesterol Education Program (NCEP) Expert Panel on Detection, Evaluation, and Treatment of High Blood Cholesterol in Adults (Adult Treatment Panel III) (2001) JAMA 285:2486-2497

3. Definition, diagnosis and classification of diabetes mellitus and its complications (1999) Report of a WHO Consultation. WHO/ NCD/NCS/99.2. WHO, Geneva

4. Balkau B, Charles MA, Drivsholm T et al (2002) Frequency of the WHO metabolic syndrome in European cohorts, and an alternative definition of an insulin resistance syndrome. Diabetes Metab 28:364-376

5. Lakka HM, Laaksonen DE, Lakka TA et al (2002) The metabolic syndrome and total and cardiovascular disease mortality in middle-aged men. JAMA 288:2709-2716

6. Shoulders CC, Naoumova RP (2004) USF1 implicated in the aetiology of familial combined hyperlipidaemia and the metabolic syndrome. Trends Mol Med 10:362-365
7. Zimmet P, Magliano D, Matsuzawa Y, Alberti G, Shaw J (2005) The metabolic syndrome: a global public health problem and a new definition. J Atheroscler Thromb 12:295-300

8. Sundstrom J, Riserus U, Byberg L, Zethelius B, Lithell H, Lind L (2006) Clinical value of the metabolic syndrome for long term prediction of total and cardiovascular mortality: prospective, population based cohort study. Br Med J 332:878-882

9. Pajukanta P, Lilja HE, Sinsheimer JS et al (2004) Familial combined hyperlipidemia is associated with upstream transcription factor 1 (USF1). Nat Genet 36:371-376

10. Huertas-Vazquez A, Aguilar-Salinas C, Lusis AJ et al (2005) Familial combined hyperlipidemia in Mexicans: association with upstream transcription factor 1 and linkage on chromosome 16q24.1. Arterioscler Thromb Vasc Biol 25:1985-1991

11. van der Vleuten GM, Isaacs A, Hijmans A, van Duijn CM, Stalenhoef AF, de Graaf J (2007) The involvement of upstream stimulatory factor 1 in Dutch patients with familial combined hyperlipidemia. J Lipid Res 48:193-200

12. Ng MC, Miyake K, So WY et al (2005) The linkage and association of the gene encoding upstream stimulatory factor 1 with type 2 diabetes and metabolic syndrome in the Chinese population. Diabetologia 48:2018-2024

13. Gibson F, Hercberg S, Froguel P (2005) Common polymorphisms in the USF1 gene are not associated with type 2 diabetes in French Caucasians. Diabetes 54:3040-3042

14. Coon H, Xin Y, Hopkins PN, Cawthon RM, Hasstedt SJ, Hunt SC (2005) Upstream stimulatory factor 1 associated with familial combined hyperlipidemia, LDL cholesterol, and triglycerides. Hum Genet 117:444-451

15. Komulainen K, Alanne M, Auro K et al (2006) Risk alleles of USF1 gene predict cardiovascular disease of women in two prospective studies. PLoS Genet 2:e69

16. Zeggini E, Damcott CM, Hanson RL et al (2006) Variation within the gene encoding the upstream stimulatory factor 1 does not influence susceptibility to type 2 diabetes in samples from populations with replicated evidence of linkage to chromosome 1q. Diabetes 55:2541-2548

17. Putt W, Palmen J, Nicaud V et al (2004) Variation in USF1 shows haplotype effects, gene:gene and gene:environment associations with glucose and lipid parameters in the European Atherosclerosis Research Study II. Hum Mol Genet 13:1587-1597

18. Dunder K, Lind L, Zethelius B, Berglund L, Lithell H (2004) Evaluation of a scoring scheme, including proinsulin and the apolipoprotein B/apolipoprotein A1 ratio, for the risk of acute coronary events in middle-aged men: Uppsala Longitudinal Study of Adult Men (ULSAM). Am Heart J 148:596-601

19. Skarfors ET, Selinus KI, Lithell HO (1991) Risk factors for developing non-insulin dependent diabetes: a 10 year follow up of men in Uppsala. Br Med J 303:755-760

20. Hsu TM, Chen X, Duan S, Miller RD, Kwok PY (2001) Universal SNP genotyping assay with fluorescence polarization detection. Biotechniques 31:560, 562, 564-568, passim

21. SeattleSNPs (2006) NHLBI Program for Genomic Applications, SeattleSNPs, Seattle, WA. Available from http://pga.gs.washing ton.edu, accessed 30 October 2007

22. Barrett JC, Fry B, Maller J, Daly MJ (2005) Haploview: analysis and visualization of LD and haplotype maps. Bioinformatics 21:263-265

23. Stephens M, Donnelly P (2003) A comparison of bayesian methods for haplotype reconstruction from population genotype data. Am J Hum Genet 73:1162-1169

24. Prentice R (1986) A case-cohort design for epidemiologic cohort studies and disease prevention trials. Biometrika 73:1-11

25. Austin MA, Edwards KL, McNeely MJ (2004) Heritability of multivariate factors of the metabolic syndrome in nondiabetic Japanese Americans. Diabetes 53:1166-1169 
26. Lin HF, Boden-Albala B, Juo SH, Park N, Rundek T, Sacco RL (2005) Heritabilities of the metabolic syndrome and its components in the Northern Manhattan Family Study. Diabetologia 48:2006-2012

27. Ford ES, Giles WH, Dietz WH (2002) Prevalence of the metabolic syndrome among US adults: findings from the third National Health and Nutrition Examination Survey. JAMA 287:356-359
28. Naukkarinen J, Gentile M, Soro-Paavonen A (2005) USF1 and dyslipidemias: converging evidence for a functional intronic variant. Hum Mol Genet 14:2595-2605

29. Goldin LR, Camp NJ, Keen KJ et al (2003) Analysis of metabolic syndrome phenotypes in Framingham Heart Study families from Genetic Analysis Workshop 13. Genet Epidemiol 25 (Suppl 1): S78-S89 